\title{
Rechtsgeschichte
}

\section{Mathias Gutmann}

Konstruktion oder Evolution der Zeit? 


\section{Abstract}

As there are no time-independent biological processes, time seems to be a biological phenomenon kat echen. This statement is true not only for developmental or evolutionary transformations, which show specific temporal and spatial patterns, but for all known physiological, genetic or molecular-biological as well as ecological or populational processes. The remarkable time dependency of all life-processes attracted the attention of biologists and philosophers - Whitehead's concept of actual entities which constitute the monad-like centrepoints of his Leibnizian universe provide a perfect example. Despite the relevance of temporal descriptions, their methodological status in the sciences is often unclear.

The aim of this paper is to present at least some of the most important non-empirical aspects of any scientific treatment of temporal phenomena. Following a constructivist approach, the introduction of temporal language particles is traced back to explicit contexts of human everyday life practices and action. "Time" is considered to represent a specific façon de parler, which allows us to deal with temporal aspects of processes and world-states, which themselves are the very subject-matter of scientific descriptions. Following this line of argument, the attempt to derive time as an epistemological precondition of subjective knowledge on grounds of biological descriptions in the perspective of an "evolutionary concept of knowledge" (Evolutionäre Erkenntnistheorie) is rejected by proving the pre-empirical, categorical status of temporal concepts. Accordingly, an evolutionary derivation of time is shown to be impossible: in contrast to naturalistic approaches, time is either to be understood as a condition of the possibility of knowledge itself (i. e. of scientific knowledge a fortiori) or it designates temporal aspects of natural processes, which are described in reference to the presupposed categories of knowledge. In both cases, »time« is neither a "natural « nor a biological object. 


\section{Konstruktion oder Evolution der Zeit?}

I. Zeit in der Biologie

Zeit ist einer jener Begriffe, für die man die Augustinische Charakterisierung gelten lassen wollte, es sei klar, was sie bedeuten, solange nicht danach gefragt werde (Augustinus Confessiones Lib. XI, I7). Die Frage aber nach dem, was »Zeit « eigentlich ist, erscheint umso berechtigter, als es insbesondere die Naturwissenschaften sind, die für sich in Anspruch nehmen, hier Antworten geben zu können. Die zu erwartenden Antworten wären danach wesentlich empirischer Natur - also direkt oder indirekt experimentell gestützt und mithin Ergebnis dieser Forschung.

Einer solchen Vermutung steht allerdings der Hinweis Janichs gegenüber, dass doch zumindest die Rede von Zeit schon verfügbar sein muss, um auch nur die Messung derselben zu ermöglichen; mehr noch stellt sich die Frage, ob nicht - zumindest anfänglich - die Messung von Zeit eine der Bedingungen der Möglichkeit von Wissenschaft überhaupt ist. In diesem letzteren Fall hätten wir in dem Ausdruck »Zeit « ein Homonym vorliegen, denn damit würde mehreres zugleich gesagt: Neben die rein wissenschaftlichen, etwa physikalischen oder biologischen Bestimmungen wären solche Klärungen zu stellen, die diesen begrifflich schon je voraus liegen (also im besten Sinne des Wortes apriorischer Natur wären). Die Homonymie gälte dann vermutlich auch innerhalb der Wissenschaften, denn selbst wenn man annimmt, dass etwa in der Biologie dieselben Zeitkonzepte verwendet werden wie in der Physik, so gilt dies doch keineswegs auch für die anderen Wissenschaften, die in diesem Zusammenhange sehr leicht ver- gessen werden, nämlich die »Geisteswissenschaften «. Wir wollen die weiteren Klärungen in mehreren Schritten vornehmen, wobei wir uns mit der Biologie ganz auf ein naturwissenschaftliches Exemplar von Wissenschaft beschränken. Nach einer kurzen sprachkritischen Klärung der relevanten Redestücke, wollen wir in einem zweiten Schritt fragen, in welchem Sinn "Zeit « ein biologisches Phänomen ist. In einem abschließenden Teil wenden wir uns schließlich Entwicklungs- und Evolutionsvorgängen zu, um den methodologischen Status zeitlicher Rede in der Biologie exemplarisch zu vergegenwärtigen.

\section{Sprachkritische Vorbetrachtung}

Peter Janich hat immer wieder auf die grundsätzlichen semantischen Probleme der Rede von Zeit hingewiesen. Die intensive Arbeit an einer sauberen, methodischen wie methodologischen Ansprüchen der Naturwissenschaften wie der Wissenschaftstheorie entsprechenden Einführung, sowohl des Ausdrucks "Zeit " selbst als auch "zeitlicher Rede« - zumindest soweit sie innerhalb des naturwissenschaftlichen Diskurses eine Rolle spielen - ist in dieser Hinsicht ein beständig weiter verfeinertes und unabgeschlossenes Projekt. Wir können zunächst feststellen, dass die grammatische Form des bestimmten Artikels ein gegenständliches Verständnis des Ausdrucks »Zeit " nahe legt, wie »der Tisch" oder »das Glas «. Der Versuch aber, jene Gegenstände aufzuzeigen, die mit dieser Rede bezeichnet werden, scheitert, denn wir werden uns vermutlich regelmäßig auf bestimmte Vorgänge beziehen, an denen wir »zeitliche Phänomene« 
beobachten. Das heißt: selbst wenn wir die nahe liegende, naturwissenschaftlich inspirierte Rede von "periodischen Vorgängen « (über die gelegentlich sogar "gleiche Dauern " definiert werden sollen, s. Janich I980: 67 f.) heranziehen, dann ist es eben nicht »die Zeit ", auf welche wir uns beziehen, sondern es sind die Vorgänge selbst, wie die (Schein-)Bewegung der Sonne über den Horizont oder das Auslaufen von Wasser aus einer Öffnung, bezüglich deren wir »zeitliche Verhältnisse " bestimmen. ${ }^{{ }^{I}}$ Schon lebensweltlich fällt dabei auf, dass wir mindestens drei Formen zeitlicher Rede nutzen, um solche Verhältnisse zu beschreiben, die wir mit Janich (I996) modale, ordinale und durative nennen wollen. Unter modalen Bestimmungen wollen wir die Rede von Gegenwart, Vergangenheit und Zukunft verstehen. Die Ausdrücke »Vergangenheit und Zukunft « beziehen sich dabei regelmäßig auf ein Tun oder Handeln, das gerade vorgeht. So kann der Vollzug einer Tätigkeit wie das Ziehen einer Linie, aber auch das Unterzeichnen eines Vertrages als »gegenwärtige« Tätigkeit beschrieben werden, während wir von einem Vertrag, der vor dem Ziehen der Linie unterschrieben wurde, als einem vergangenen Ereignis sprechen und von dem morgen zu unterzeichnenden als zukünftigem. Die Rede von Vergangenheit, Gegenwart und Zukunft folgt der adjektivischen resp. adverbialen Redeform durch Substantivierung.

Ordinale Verhältnisse bezeichnen wir mit Ausdrücken wie »früher " oder »später «. Sie legen Reihenfolgen von Handlungen oder Handlungsschritten sowie von Handlungsergebnissen und -folgen fest, und es sind die Vollzüge solcher Handlungen - wie das Ziehen einer Linie oder das Unterzeichnen eines Vertrages -, bezüglich deren wir feststellen, was früher oder später erfolgte. Mit »davor« und »danach« bzw. »zu- gleich « können wir entsprechend gerade solche Verhältnisse bezeichnen, womit sich schon hier zeigt, dass zeitliche Ausdrücke regelmäßig auch in anderen Zusammenhängen fungieren, etwa als räumliche oder logische (man denke exemplarisch an den Kollegen, der sich in der Postschlange nach uns anstellte, oder die Aussage, die nach gewissen Regeln aus gegebener Prämisse folgt). ${ }^{2}$

Mit der durativen Verwendung zeitlicher Ausdrücke bezeichnen wir ferner die Aspekte der Dauer. Ausdrücke wie "gleichlang " oder polar-konträre Ausdrücke wie »länger-kürzer « dienen dabei dem Vergleich von Vorgängen z. B. wieder Handlungen - hinsichtlich eines vereinbarten Standards. Die Reihenfolge, in der zeitliche Ausdrücke vorgestellt wurden, kann zugleich als eine methodische verstanden werden, denn wir müssen z. B. über modale Redestücke schon verfügen, bevor wir (sowohl zeitlich wie systematisch) ordinale oder durative verwenden können.

Der Ausdruck "Zeit « schließlich kann, Janich folgend, als Reflexionsterminus eingeführt werden. Das heißt es handelt sich um ein Redestück, das sich seinerseits auf (in diesem Fall) zeitliche Ausdrücke bezieht. Wir können darunter eine offene Liste von Ausdrücken verstehen, die jederzeit verlängert werden kann, wenn weitere Bestandteile aufzunehmen zweckmäßig erscheint. Der durch den bestimmten Artikel erweckte Eindruck eines gegenständlichen Referenten entpuppt sich also in der vorgestellten Rekonstruktion als grammatischer Schein. Zeit als Substantivierung zusammenfassender Rede über zeitliche Verhältnisse fungiert vielmehr in den angezeigten und in den daran angeschlossenen Sprachspielen auf höchst unterschiedliche Weise. Dies wird besonders deutlich, wenn wir den Übergang zur Zeitmessung vollziehen.

$\mathrm{Zu}$ den Definitionsvorschlägen für Zeitmessstandards und den dabei regelmäßig auftretenden Schwierigkeiten s. JANICH (I980) 37 ff.

2 Wir finden hier zudem eine Geltungsasymmetrie von räumlicher und zeitlicher Rede, die die letztere an erstere bindet, was sich exemplarisch an Ausdrücken wie "Zeitpunkt " oder "Zeitraum « zeigt -

일 dazu auch CASSIRER (I923I929). 
3. Von der Lebenswelt zur Zeitmessung

Die bisherigen Überlegungen bewegten sich alle noch innerhalb lebensweltlicher Redeformen. Doch schon hier trat das Problem der Zeitmessung auf. Denn wenn Dauern verglichen werden sollen, und sei es noch so vorläufig, müssen Verfahren des Zeitvergleiches bereitstehen. Der Übergang zum Uhrenbau konfrontiert mit einigen bekannten definitorischen und technischen Problemen. So wäre es selbstverständlich möglich, gerade den üblichen Weg zu gehen und einfach auf »natürliche periodische Vorgänge« zu verweisen. Diese Lösung versagt aber auffällig, denn zur Auswahl jener Vorgänge, denen das Prädikat "gleichmäßig « zukommen soll, benötigen wir schon eine Messmöglichkeit (zumindest in einem ganz elementaren, praktischen Sinne) - wir wären also mit einem echten hysteron-proteron konfrontiert. Dieses lässt sich vermeiden, wenn man die Frage nach der Messung von Zeit, die ja suggeriert, es gebe einen mit Zeit bezeichneten Gegenstand, an den dann Messgeräte (nämlich Uhren) heranzutragen wären, transformiert in die Frage nach dem Referenten der zeitlichen Redeformen, für deren Vergleiche wir Uhren verwenden. Folgen wir der handlungstheoretischen Vorgehensweise, dann gelingt der Vergleich von Dauern durch die Lösung des technischen Problems der Erzeugung oder Auszeichnung normierter Standardbewegungen. Die Geräte, ${ }^{3}$ welche bestimmte Kriterien zu erfüllen haben, um die geforderten Bewegungen bereitzustellen, wären dann gerade jene von uns schon als Messgeräte apostrophierten Uhren. Eine Uhr ist in dieser Beschreibung zunächst vor allem ein Apparat zur Erzeugung geregelter Bewegungen. Aus den Herstellungsnormen, mit denen wir auch die Funktionsfähigkeit der Geräte sicherstellen, d.h. technisch erzwingen, er- geben sich die entscheidenden Eigenschaften der Uhren. Diese Eigenschaften kommen Uhren also nicht natürlicherweise $\mathrm{zu}$ (wiewohl selbstverständlich Naturvorgänge als Uhren genutzt werden können; siehe unten), sie ergeben sich vielmehr aus den Zwecken, für deren Erreichung Uhren die probaten Mittel sind. Für den Vergleich von Bewegungen, die von zwei oder mehreren solcher Apparate hergestellt werden, lassen sich »Homogenitätskriterien « definieren, über die dann auch die Rede von "gleichförmig" (ideativ) eingeführt werden kann:

»Entsprechend den methodologischen Erörterungen [...] muss jetzt noch ein Ideationsverfahren angegeben werden. Zu diesem Z Zweck wird das chronometrische Homogenitätsprinzip aufgestellt:

$$
\text { sev s'ev a }(s, v) a\left(s^{\prime}, v\right)
$$

d.h. zwei Stellungen s und s' aus dem Vorgang v (im Gerät G) sind ununterscheidbar, >a $<$ sind dabei Aussagen, in denen keine freien Variablen vorkommen dürfen, wohl aber unter Quantoren gebundene. Durch eine in diesem Sinne zulässige Ersetzung von a durch eine Aussage, die mithilfe des Vokabulars der uhrenfreien Kinematik möglich ist, entsteht aus dem Homogenitätsprinzip (H) der Homogenitätssatz H. Beschränkt man sich auf diejenigen Aussagen A, die aus $\mathrm{H}$ logisch folgen, spricht man also invariant bezüglich Realisierungsmängeln über gleichmäßige Vorgänge, so soll zur terminologischen Kennzeichnung dieses Übergangs an die Stelle von sgleichmäßig< der Ideator >gleichförmig < treten. [...] Ein Gerät, an dem sich ein Punktkörper (Zeiger) gleichförmig bewegt, heiße Uhr.« (Janich I980: 202)

Das eigentliche Problem des Baues einer ersten Uhr besteht nun gewissermaßen darin, diese nicht als Zeitmesser, sondern als Apparat
3 Die Kriterien gelten auch für natürliche Vorgänge; erst durch die Lösung der Probleme, die mit der Gleichförmigkeit der Bewegungen verbunden sind, kann von diesen ja gesagt werden, dass und $o b$ sie gleichmäßig sind. Da nur Geräte der (im Ideal vollständigen) Kontrolle des Menschen unterliegen, ist es daher auch sinnvoll, gerade bei ihnen zu beginnen - dies darf aber nicht mit einer historischen

Aussage verwechselt werden. 
zur Erzeugung von - nach explizierbaren Kriterien angebbaren - gleichförmigen Bewegungen zu beschreiben. Eine solche Beschreibung kann geliefert werden z. B. für eine nach »Walzenprinzip « funktionierende Apparatur (s. Janich I988). Der methodologisch entscheidende Schritt besteht darin, dass wir die Frage nach der »richtigen « Zeitmessung übersetzen können in die Frage nach dem Bau störungsfrei funktionierender Apparate zur Erzeugung von Bewegungen. Diese Bewegungen müssen selbstverständlich nicht periodisch sein - es sind ja schon historisch am Beispiel der Klepshydra oder der lebensweltlich gut bekannten Sanduhr Exemplare zur Lösung des zugrunde liegenden technischen Problems angebbar, die auf nicht-zyklischen Vorgängen basieren. Der Gleichlauf wird in jedem Fall durch technische Parameter kontrolliert, die ihrerseits "zeitunabhängig " sind. ${ }^{4}$ Bedeutet dies alles nun, dass es »in der Natur « keine Zeit »gibt «?

\section{Ist Zeit ein natürliches Phänomen?}

Die Beantwortung dieser Frage hängt selbstverständlich von der Verwendung des Ausdruckes »Natur « ab. Dieser wäre zunächst allenfalls im Sinne eines Titelwortes zu verstehen, dessen einzelne Einträge - so wie im Falle zeitlicher Ausdrücke - Familienähnlichkeiten aufweisen, denn immerhin gehören dazu so unterschiedliche Gegenstände wie »das Wesen einer Sache «, »Gegenstand naturwissenschaftlicher Beschreibungen «, »der Gegensatz zu künstlich, oder vom Menschen verfertigt « etc. ${ }^{5}$ Wir wollen unsere Klärungen auf den Bereich naturwissenschaftlich beschriebener Gegenstände und dies zudem exemplarisch auf biologisch beschriebene Gegenstände beschränken.
Es ist nahe liegend, unter Zeit einen biologischen Gegenstand zu sehen, in einem doppelten Sinne:

I. Zeit bezeichnet etwas »an biologischen Vorgängen «.

2. Zeit ist Ergebnis eines biologischen Vorganges, der seinerseits Bedingung der Möglichkeit von Erkenntnis (etwa von Zeit) ist.

Im ersten Fall wäre der Ausdruck »biologische Zeit « eine abkürzende oder sogar metaphorische Redeweise über biologische Vorgänge, die wir in einer ausgezeichneten Hinsicht zeitlich, d.h. unter Nutzung zeitlicher Ausdrücke beschreiben. In diesem Fall gäbe es keinen Grund für die Vermutung, dass die Mittel der Beschreibung selbst - zumindest in einem ersten Schritt wieder Resultate biologischer Beschreibungen sind oder unter konstitutiver Nutzung entsprechender Sprachstücke zustande kamen. Es träfe dann die auf den ersten Blick seltsam anmutende Aussage des Aristoteles zu:

"Denn eben das ist die Zeit: Die Messzahl von Bewegungen hinsichtlich des >davor >danach . Also: Nicht gleich Bewegung ist die Zeit, sondern insoweit die Bewegung Zahl an sich hat (gehört sie zu ihr). Ein Beleg dafür: Das >mehr< oder `weniger entscheiden wir mittels der Zahl, mehr oder weniger Bewegung mittels der Zeit; eine Art Zahl ist also die Zeit. « (Aristoteles, Physik IV, I I 2 I 9b)

Unbestreitbar ist nun die Tatsache, dass viele (und in gewisser Hinsicht alle) biologische(n) Vorgänge sich zeitlich vollziehen. Für einige dieser Vorgänge wird zudem die Bezeichnung einer »biologischen Uhr « gewählt, um die Zuverlässigkeit zumindest der Form dieser Vorgänge zu betonen. Sehr gute Beispiele hierfür sind etwa die Tagesperiodizität der Blattbewegungen von Vicia faba, der Motorialaktivität der Zauneidechse oder die Schlüpfrhythmen von Drosophila (hier-

\footnotetext{
$4 \mathrm{Zu}$ den vor allem kulturell-historischen Hintergründen des Baues von Uhren mit rundem Ziffernblatt s. JANICH (I980).

$5 \mathrm{Zu}$ den damit verbundenen methodologischen Problemen s. GutManN (2002), JANICH (I997).
} 
zu Mletzko und Mletzko I985; zu Einzelstudien s. etwa Tembrock et al. I986). Doch auch für größere Skalen stehen solche biologischen Uhren bereit, wenn wir etwa an "molekulare Uhren " denken, die für die Skalierung evolutionärer Vorgänge relevant sind. Diese "molecular clocks « gehen zunächst auf eine Beobachtung zurück, die an Austauschraten von Aminosäuren bei Hämoglobin und Cytochrom c gemacht wurden. Diese erwiesen sich - zumindest auf den ersten Blick in vielen der untersuchten Mammalier-Linien als mehr oder minder gleichförmig. Eine molekulare Uhr wäre nun gerade dann identifizierbar, wenn sich - bezogen auf bestimmte Proteine - die Austauschraten über die Zeit als konstant in vielen (oder möglichst allen) Linien erwiesen. Damit ließen sich nämlich evolutionäre Zusammenhänge - etwa die Bestimmung von Divergenz-Zeiten - gut rekonstruieren, was in die Formulierung von Kimuras evolutionärem Gesetz einmündet (Graur und Li 2000: I40). Gegen die Nutzung von Austauschraten als Uhren lassen sich einige Einwände formulieren, die in zwei Gruppen zu unterscheiden sind:

I. Empirische Einwände. Diese richten sich gegen die Grundannahmen der Uhren-Hypothese, etwa durch Nachweis der Veränderung von Raten wie im Falle des Insulins, der Schwierigkeit, die Veränderung von Funktions-Regimen nachzuweisen oder auszuschließen; hinzu käme etwa der Ausschluss von ratenverändernden Effekten wie Metabolismuswerte, der unterschiedlichen Effizienz der DNA-Reparatur-Systeme, oder dem time-generation-effect (s. Graur und Li 2000: I 5 I).

2. Methodologische Einwände. Diese lassen sich in zwei Gruppen einteilen, wobei der eine Einwandtyp sich gegen neutral-selektionistische Annahmen richtet, die durch die molecularclock-Hypothese eine gewisse Stützung erfuhren.
Für uns interessanter ist aber die zweite Gruppe von Einwänden, die danach fragen, inwieweit es sich bei den Befunden überhaupt um eine »Uhr « handelt. Immerhin dürfte selbst bei den Befürwortern unstrittig sein, dass die Universalität derselben eingeschränkt ist:

"Since the assumption of rate constancy is violated even within Mammalia, a truly universal molecular clock that applies to all organisms cannot be assumed to exist. Indeed, the substitution rate among distantly related organisms has been invariably shown to vary to a greater or lesser extent. For example. It has been estimated the rate of substitution in Drosophila species is 5-IO times faster than the rate in most vertebrates, and this difference holds even for the most conservative portions of the genome [...]. Therefore, extreme caution must be exercised when using the molecular clock assumption to infer times of divergence, more so when distantly related species are concerned." (Graur und Li 2000: I 5 I)

Diese mangelnde Universalität ist nicht nur (wiewohl sicher auch) ein empirisches Problem, sondern ein methodologisches. Denn um die Austauschvorgänge bei Molekülen als Uhren im Sinne einer Standarddauer für evolutionäre Vorgänge nutzen zu können, müssen die Austauschvorgänge auf weitere Vorgänge bezogen werden, an denen evolutionäre Übergänge (etwa innerhalb der Mammalier-Reihe oder auch zur Mammalier-Organisation hin) orientiert werden können. Diese "Kalibrierung " findet etwa an geologischen resp. paläontologischen Befunden statt:

"The use of molecular clocks is based on the premise that many molecular differences among taxa are neutral mutations that accumulate at a relatively constant rate when averaged over geological time $[. .$.$] . Therefore, the larger the mo-$ 
lecular difference, the earlier the inferred divergence date. The irony, at least as far as paleoanthropologists are concerned, is that molecular clocks must ultimately be calibrated against the fossil record." (Conroy I997: I2I)

Es muss also jedenfalls ein (sedimentologisches sowohl als paläontologisches) Wissen investiert werden, um molekulare Austauschraten in Aussagen über Zeitdifferenzen oder sogar absolute Zeitpunkte übersetzen zu können. Das Wissen über diese Zeitpunkte darf daher logisch nicht wieder von dem molekularbiologischen Wissen abhängen, das damit begründet werden soll, da sich sonst Zirkel einstellen (zum Rekonstruktionsproblem in evolutionärer Hinsicht s. unten).

Wie die Rekonstruktion der Rede über molekulare Uhren zeigt, handelt es sich auch bei den anderen angeführten Beispielen biologischer Uhren um »Uhren « nur insofern, als sie als solche beschrieben und - möglicherweise - gehandhabt werden. Mit anderen Worten: Die biologischen Vorgänge können genau dann als Uhren in dem oben bestimmten protophysikalischen Sinne gelten, wenn für die Vorgänge, die hier experimentell erfasst werden, jene Kriterien gelten, die für Uhren im handlungstheoretischen Sinne als relevant ausgezeichnet wurden. In dieser Beschreibung von Vorgängen als Uhren ist es ersichtlich gleichgültig, welcher Art die Vorgänge selbst sind: Es können Strahlungen beim Übergang zwischen Hyperfeinstrukturniveaus des Grundzustandes von I 23 Cs ebenso sein wie Blattbewegungen von Pflanzen. Das Anfangsproblem, das mit Janich handlungstheoretisch gelöst werden kann, ist aber auf diese Weise gerade nicht zu lösen, es muss vielmehr schon als gelöst unterstellt werden, um überhaupt sagen zu können, es handele sich bei einem »natürlichen « Vorgang um einen Standard für ein zeitliches Phänomen.
Damit ist die Aussage, "Zeit « sei Ergebnis biologischer Vorgänge (auf die es uns ja hier vor allem ankam), eine nur metaphorische Beschreibung dafür, dass biologische Vorgänge als Uhren ausgezeichnet wurden. Diese bringen Zeit so wenig hervor wie jede andere Uhr.

5. Ist Zeit ein erkenntnisbiologisches Problem?

Eine andere - und in den Folgen für die Geltung der resultierenden zeitlichen Aussagen wesentlich tiefer greifende - Herangehensweise bestünde nun darin, zu vermuten, dass Zeit (und damit verbunden zeitliche Verhältnisse) selbst direkte oder indirekte biologische Leistung jener Lebewesen sei, die versuchen, "Zeit « zu verstehen. Diese Vermutung könnte ihre empirische Anfangs-Stützung in Beobachtungen zu circadianen Oszillationen finden, die etwa durch die rhythmische Ausschüttung des Melatonin durch das Pinealorgan bei einigen Vögeln oder Nagern zustande kommen (s. Winfree I986). Überträgt man solche Beobachtungen auf den Menschen, so ließe sich z. B. konstatieren, dass Zeit durch die enge Verknüpfung mit dem neuronalen Apparat höherer Säuger tatsächlich ein - von diesen gleichsam selbst hervorgebrachter - Naturvorgang sei. Das Konzept einer hier anzuschließenden Evolutionären Erkenntnistheorie (EET) liefe gerade darauf hinaus, Zeit als Produkt biologischer Systeme zu begreifen, ein Gedanke, dessen mögliche erkenntnistheoretische Implikationen wir - aller Unterschiede zu aktuellen Ansätzen ungeachtet - mit Uexküll wie folgt bestimmen können:

»Die Aufgabe der Biologie besteht darin, die Ergebnisse der Forschungen Kants nach zwei Richtungen zu erweitern: I. Die Rolle unseres Körpers, besonders unserer Sinnesorgane und 
unseres Zentralnervensystems mit zu berücksichtigen und 2. Die Beziehungen anderer Subjekte (der Tiere) zu den Gegenständen zu erforschen." (Uexküll I973: 9)

Deutlich über die Formulierung Uexkülls hinausgehend (wiewohl dessen Antidarwinismus durchaus teilend), fasst Lorenz eine evolutionäre Erkenntnistheorie mit direktem Bezug auf transzendentale Aprioris wie folgt:

»Wenn Hartmann, wie aus den zitierten Sätzen genugsam hervorgeht, fest voraussetzt, daß die Verstandeskategorie zugleich Gegenstandskategorie ist, und wenn er, wie er es tatsächlich tut, darauf seine Überzeugung von der Existenz und der relativen Erkennbarkeit der Außenwelt gründet, so ist er in seiner erkenntnistheoretischen Grundhaltung derjenigen des hypothetischen Realismus auf das nächste verwandt, für den die Kategorien und Anschauungsformen des menschlichen Erkenntnisapparates ganz selbstverständlich etwas im Laufe der Stammesgeschichte Gewordenes sind, das auf die Gegebenheiten der außersubjektiven Wirklichkeit in analoger Weise und aus analogen Gründen >paßt $<$ wie der Pferdehuf auf den Steppenboden oder die Flußflosse ins Wasser." (Lorenz I973: $56 \mathrm{f}$.)

Die Kantischen Formen des Apriori (hier unvernehmlich der Unterscheidungen von Anschauungsformen und Kategorien) würden also als individuelle Erscheinungen eines stammesgeschichtlichen Aposteriori aufzufassen sein (dazu weiterführend etwa Vollmer I987: I 8 ff.).

Ähnliche - und dabei darwinistische Einsichten integrierende - Bestimmungen finden sich in der Literatur zahlreich (man denke nur an Simpsons danebengreifenden - und daher toten - Affen). Unterscheiden wir zum Zwecke der Untersuchung dieses Arguments wieder empirische von methodologischen Einwänden und konzentrieren uns auf die letzteren, dann muss festgestellt werden, dass das zentrale Anliegen einer EET in der Bindung der Geltung von Aussagen über etwa Raum und Zeit (sowie möglicherweise andere »Erkenntnisvoraussetzungen «) an die Geltung biologischer und häufig evolutionsbiologischer Aussagen besteht; eine solche Verknüpfung ist selbstverständlich immer zulässig. Sie hat allerdings eine für das Gesamtunternehmen EET bedrohliche Konsequenz, die sich exemplarisch an der Verwendung der Ausdrücke »wahr« und »falsch« darstellen lässt. Wird nämlich für diese Unterscheidung ebenfalls (wie es konsequenterweise geschehen müsste) die Bindung an geltendes biologisches Wissen gefordert, dann folgt ein Dilemma, das sich aus der trivialen Vermutung ergibt, dass das investierte biologische Wissen mindestens geltendes Wissen sein soll, denn

I. entweder ist die Geltung der zu investierenden biologischen Aussagen selbst unabhängig von der wahr/falsch-Unterscheidung. Wir hätten es damit notwendig nicht mehr mit empirischem Wissen zu tun,

2. oder die Empirizität dieses Wissens wird ebenfalls zugegeben.

Im ersten Fall wäre die Begründung der $\mathrm{zu}$ investierenden biologischen Aussagen nicht mehr (natur-)wissenschaftlich zu leisten. Dies führt dazu, entweder Evolutionstheorie als eine grundsätzlich, als Theorie von anderen empirischen Theorien unterschiedene Theorie zu verstehen, oder es führt zu einer schlicht dogmatischen Feststellung der (dann notwendigerweise beschreibungsunabhängigen) Existenz des $\mathrm{Na}$ turvorganges »Evolution «. Diesen Weg beschreitet etwa Oeser, wenn er den Status von Evolutionstheorie wie folgt bestimmt:

"Eine solche universale Theorie der Evolution ist ex definitione nicht bloß eine Angelegen- 
heit der Biologen. Sie ist vielmehr eine allgemeine, direkt nicht falsifizierbare Hintergrundtheorie, die jedoch in allen ihren abgeleiteten Teilen überprüfbar ist. "(Oeser I987: 53)

Mit dieser Exemption der Evolutionstheorie kollidiert allerdings inhaltlich die schlichte Tatsache, dass es nicht »eine « Evolutionstheorie gibt (entgegen der wiederholten Behauptung vor allem seitens der Vertreter der Synthetischen Evolutionstheorie; siehe etwa Kutschera 2006). ${ }^{6}$ Methodologisch wird zumindest nahe gelegt, unter Evolutionstheorie keine im üblichen Sinne empirische Theorie zu sehen; diese Sonderstellung ist aber lediglich eine Folge der Argumentstruktur der Evolutionären Erkenntnistheorie selbst. Da Evolutionstheorie als jenes Wissen investiert werden muss, bezüglich dessen ontogenetische Aprioris als phylogenetische Aposterioris sich beschreiben lassen, darf dieses Anfangswissen selbst nicht noch einmal (empirisch) zur Disposition stehen, was in das oben angezeigte Dilemma zurückführt, die Herkunft dieses investierten Wissens aber unbestimmt lässt.

Der zweite Fall führte uns direkt in einen Regress, denn nun wäre die jeweils notwendige wahr/falsch-Unterscheidung als empirisch behauptet und damit von weiterer Forschung abhängig gemacht, für die dasselbe wiederum gälte. Ein weiteres Ungemach tritt hinzu, indem wir nun nämlich auch die wahr/falsch-Unterscheidung außerhalb empirischer Zusammenhänge (etwa in Formal- oder Idealwissenschaften) als letztlich empirisch zu bestimmen hätten.

Dies zeigt exemplarisch Uexkülls Vermutung, es ließe sich die Dreidimensionalität des Raumes aus der Stellung der Bogengänge zueinander "ableiten « (Uexküll I973: 32 ff.). Eine radikale und in dem Sinne des aufgezeigten Problems konsequente Weiterführung findet sich bei Lorenz:
»Es erscheint mir als eine abstruse Annahme, daß alle diese, so offensichtlich im Dienste arterhaltender Leistungen und in Anpassung an reale Gegebenheiten entstandenen Organe (das Labyrinth und der Utriculus, MG) und ihre Leistungen nicht mit unserer apriorischen Anschauungsform des Raumes zu tun hätten. Es erscheint mir vielmehr als selbstverständlich, daß sie der Anschauungsform des dreidimensionalen seuklidischen $<$ Raumes zugrunde liegen, ja, daß sie in gewissem Sinne diese Anschauungsformen sind. Wir wissen von den Mathematikern, daß andere mehrdimensionale Arten von Raum denkmöglich sind, und von den Relativitätstheoretikern und Physikern, daß es mindestens vier Dimensionen des Raumes nachweislich gibt. Anschaulich erleben können wir aber nur jene einfachere Version, die unsere arteigene Organisation der Sinnesorgane und des Nervensystems in Erfahrung bringt «." (Lorenz I973: 22)

Unabhängig von der Richtigkeit der Rede über die »vier Dimensionen « des Raumes ist diese Empirisierung des Euklidischen Raumes als natürlichem Aspekt der evolutionären Anpassung an die »realen Gegebenheiten " aber mit methodologischen Einwänden konfrontiert. Denn um wissen zu können, dass die Dreidimensionalität den realen Gegebenheiten entspricht, müssen wir genau diese Entsprechung beurteilen können. Dies gelingt aber ersichtlich nur, wenn wir entweder ein unmittelbares Wissen von diesen Gegebenheiten hätten oder aber die Rede über die Unterscheidung von dreidimensionalen und nicht dreidimensionalen Gegebenheiten schon vorliegt. Im ersten Fall evozierten wir wieder den oben schon angezeigten Dogmatikvorwurf, im zweiten Fall wäre entweder zuzugeben, dass das Wissen um die Einführung dreidimensionaler Gegebenheiten unabhängig von biologischem Wissen ist (es wäre letzterem dann

6 Zu nicht- und anti-darwinistischen Evolutionstheorien siehe u. a. Levit et al. (2005). 
wieder in genauer Weise vorausgesetzt), oder es müsste seinerseits wieder auf biologisches Wissen zurückbezogen werden, was zum einen der Prämisse Oesers von der »Nicht-Falsifizierbarkeit " widerspricht, zum andern aber das Regress-Argument (jetzt in Form vorgelagerter biologischer Erläuterungen des Wissens um die Dreidimensionalität von Gegebenheiten) evozierte.

Ganz unabhängig von der Frage, ob es nicht gerade dem im engeren Sinne naturwissenschaftlichen Anspruch evolutionstheoretischer (und -biologischer) Forschung widerspricht, gegen Falsifikation immunisiert zu werden (hier hätte ein gut geführtes ID-Argument leichtes Spiel), ist daher das Wissen um die Dreidimensionalität des Raumes unabhängig von biologischem - und damit a fortiori evolutionsbiologischem Wissen zu gewinnen. Wollen wir hier nicht mit »Evidenz-Überlegungen « o. ä. begründen, dann erscheint Janichs Vorschlag als sinnvolle normativistische Alternative zur deskriptivistischen Vermutung, Dreidimensionalität sei eine Eigenschaft der Natur oder der natürlichen Einrichtung (menschlicher) Sinnesorgane. Diese normativistische Alternative betont den Herstellungsaspekt von Formen an Körpern; es lässt sich zeigen, dass Dreidimensionalität (und in diesem Sinne Euklidizität) eine (durch die Zugrundelegungen der Herstellungsvorschriften für bestimmte Formeigenschaften an Körpern) erzwingbare Eigenschaft nicht der Natur, sondern letztlich unserer (Raum-)Messinstrumente ist. Unter Aufweis, dass jeder Körper genau dreimal strikt fortgesetzt halbiert werden kann, lassen sich sogar die Herstellungsanweisungen für euklidische Verhältnisse explizit angeben (s. Janich I989). Dies hat erkenntnistheoretisch tiefgreifende Folgen, die wir mutatis mutandis auf das Zeitproblem übertragen können:
»Der snotwendige< wenigstens praktisch apriorische Charakter der Dreidimensionalität hängt also damit zusammen, dass für alle räumlichen Erfahrungen in der Welt ein Ordnungsschema mit den Grundbegriffen der euklidischen Geometrie technisch erfolgreich angewendet wird. Daran hat weder die Favorisierung nichteuklidischer Geometrien in Teilen der modernen Physik historisch etwas geändert, noch könnte sich aus einer - für sich gerechtfertigten - Anwendung nichteuklidischer Geometrien in systematischer Hinsicht etwas ändern. Die Dreidimensionalität des Raumes ist mit andern Worten die Kulturleistung des Menschen, die Welt durch Erfindung und technische Herstellung von Ebenen und rechten Winkeln auf eine höhere Stufe der Erkennbarkeit zu heben. «(Janich I989: 218)

Wir können diese - auf den Raum als Apriori jeder empirischen räumlichen Betrachtung bezogene - Einsicht nun auch für die Zeit in Anwendung bringen. Der erkenntnistheoretische Vorteil besteht darin, dass wir zur Etablierung von Zeit als Rede über zeitliche Verhältnisse, die ihrerseits gebunden sind an menschliches Herstellungswissen, keinerlei biologisches Wissen in Anspruch nehmen müssen. Umgekehrt wäre aber Biologie als Wissenschaft überhaupt erst möglich, nachdem die Zeitmessstandards schon etabliert sind. Diese Lösung des Zeitproblems erübrigt die erkenntnistheoretischen Einwendungen gegen eine EET, wie wir sie oben vortrugen. Allerdings hat sie Folgen für unser Verständnis von Zeit als Naturgegenstand:

I. Es wäre nicht Zeit der Gegenstand naturwissenschaftlicher Untersuchung, sondern die Bestimmung zeitlicher Verhältnisse an Gegenständen der wissenschaftlichen Betrachtung.

2. Solchen Gegenständen wären dann erst empirische Untersuchungen zeitlicher Verhält- 
nisse anzuschließen, z.B. von Bewegungen im physikalischen Sinne (als Relativbewegungen von Körpern etwa), aber auch in dem oben explizierten biologischen Sinne. So ließen sich Lokomotion, Motilität, Mobilität ebenso zum Gegenstand der messenden und vergleichenden Betrachtung nehmen wie physiologische Abläufe (man denke an den reduktiven oder oxidativen Pentosephosphatzyklus), Entwicklungsvorgänge oder evolutionäre Transformation.

Naturgegenstand würde also Zeit nur insofern, als es sich um Aussagen über zeitliche Verhältnisse an naturwissenschaftlich beschriebenen Vorgängen handelte. Die Besonderheit der empirischen Betrachtung von Zeit in der hier vorgelegten Rekonstruktion lässt sich aufzeigen, wenn wir abschließend auf einen Unterschied eingehen, der üblicherweise als ein Skalierungsproblem aufgefasst wird: bei dem Vergleich von Entwicklungsvorgängen auf der einen Seite und evolutionären Vorgängen auf der anderen.

\section{Zur Logik der Entwicklung}

Mit der Rede von »entwickeln « tritt uns ein Wortfeld entgegen, innerhalb dessen sich sowohl substantivische, adjektivische wie verbale Verwendungen ausmachen lassen. Diese Verwendungsweisen zeigen ihrerseits jeweils große Varianz. Allen gemeinsam ist immerhin der Bezug auf »Veränderungen «. »Entwickeln « als »Verändern « aufgefasst wäre damit zu ergänzen hinsichtlich des Gegenstandes, des Anfanges, des Ergebnisses und des Modus' der Veränderung, seine Bedeutung durch strenge Kontextualisierung zu ermitteln bzw. durch Indizierung anzugeben. Grundsätzlich kann die irreflexive von der reflexiven Verwendungsweise unterschieden werden: ${ }^{7}$
I. Bei der irreflexiven Verwendung des Verbs »entwickeln « (» Watt entwickelte den Prototyp der modernen Dampfmaschine mit Fliehkraftregler «) kann eine vollständige Explikation mit Bezug auf Herstellungs- oder Produktionsformen vorgenommen werden. Es wären also der Ausgangszustand (etwa die Newcomen-Dampfmaschine), der Veränderungsmodus (etwa eine spezifische Optimierung), die Beurteilungskriterien (etwa die Leistungskriterien) anzugeben. Je nachdem, ob von einem beschriebenen Endzustand her die Vorläufer angegeben werden oder von einer zu verändernden Maschine die nachfolgenden zum gewünschten Zustand führenden Schritte, lässt sich ferner die progressive von der regressiven Redeform unterscheiden. Zwar wird die Beantwortung der Frage, welche Vorläufer zu einem bestimmten Gegenstand führten, in der Regel als Ursprungserzählung berichtet, d.h. von einem zeitlichen Früher zu einem zeitlichen Später. Die hier als »Anfangszustand « gesetzte Beschreibung ist aber in der methodischen Reihe der Abfolge der »Endzustand « et vice versa. Die tatsächliche Erstellungsreihenfolge ist somit keine zeitliche, sondern zunächst eine methodische, die dann in der Berichtsperspektive in eine zeitliche umgedeutet wird.

2. Zur Verdeutlichung der reflexiven Verwendung denke man exemplarisch daran, dass »das Huhn sich aus dem Ei entwickle«. Im Gegensatz zur irreflexiven Redeform ${ }^{8}$ zeigt das reflexive »sich" hier einen bestimmten Bezug auf einen Verlauf an. Eine Beschreibung der Veränderungen während dieses Verlaufes gelingt allerdings schon lebensweltlich - ohne Nutzung einer explizit normierten Fachsprache - bzw. technisch im Sinne des Züchtungswissens. In diesem Sinne ist das Ei das Edukt, das Huhn das Produkt, ${ }^{9}$ während die reflexive Wendung den Unterschied zur irreflexiven Verwendung im

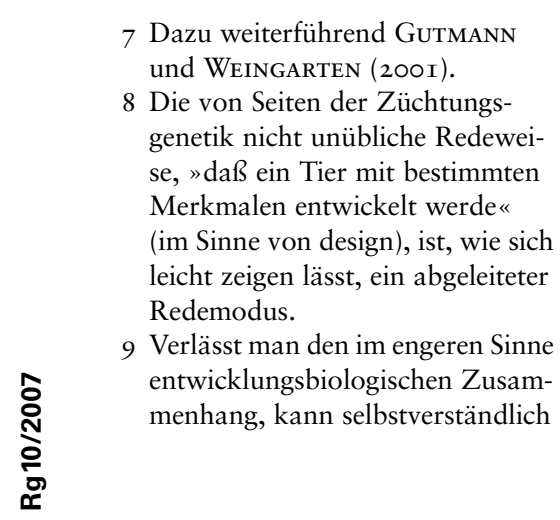

Konstruktion oder Evolution der Zeit? auch das Ei als Produkt beschrieben werden. 
Produktionsvorgang bezeichnet, insofern dieser auch ohne weiteres Zutun des Menschen verläuft. ${ }^{\text {10 }}$ Hier bezeichnet die »progressive « Rede über Veränderung also die Herstellung von Lebewesen mit bestimmten Eigenschaften als Handlungsziel, welches dann unter Verwendung einschlägiger Verfahren zu realisieren ist. Auch die »regressive « Rede ist ganz analog zum Herstellungsverfahren belegt und gibt Auskunft über den bisherigen Verfahrensgang (im Beispiel also die Züchtungslinie). Formal entspricht dies aber dem irreflexiven Modus mit dem Unterschied, dass das Produktionsverfahren gegenüber den oben einschlägigen gewisse Eigentümlichkeiten aufweist. Im Gegensatz zur irreflexiven handelt es sich bei der reflexiven Redeform um eine »eigentlich" metaphorische Wendung. Die Explikation gelingt nur unter Bezug auf Wissen, welches an Produktionsformen im irreflexiven Modus gewonnen wurde. Nimmt man nämlich das Verb »entwickeln « als Aktivum und schreibt dieses dem Huhn zu, welches aus dem Ei entstehe, dann ist der Verweis auf HerstellungsHandlungen der entscheidende explikative Hintergrund. $^{\text {II }}$

Der grundsätzliche Unterschied beider Verwendungen von » entwickeln « besteht im methodologischen Primat der irreflexiven Form. Dabei ist der Modus der Veränderung (über den dann entwickeln spezifiziert wird) jeweils »als Produktion oder Hervorbringung « explizit anzugeben. Dies gelingt unter direktem Bezug auf menschliches Herstellungs- oder Manipulationswissen. Wird die Züchtungspraxis als methodischer Anfang der (Evolutions-)Biologie gewählt, wie dies bei Darwin zumindest der Form nach geschieht, ${ }^{\text {I2 }}$ so können sowohl Züchtungsverfahren als auch die in diesem Rahmen verwandten Beschreibungen für biologische Zwecke genutzt werden.
7. Entwicklung als Verlauf

im hypothetischen Modus

Folgt man der konstruktiven Lesung der Darwinschen Züchtungsmetapher, dann lässt sich in der irreflexiven Form über die züchterische Veränderung von Lebewesen (durch den Züchter) nach Maßgabe menschlicher Praxen sprechen. Regressiv kann ferner die bisherige Zuchtlinie als eine Abfolge von »Antezedenten « der "gegebenen « Züchtungsformen angeführt werden. Nun kann zur reflexiven Form (etwa »die Mammalia haben sich aus höheren Theropoden entwickelt «) übergegangen werden. Das gelingt, indem das Züchtungswissen als Modell für die Rekonstruktion möglicher Vorläufer rezenter Formen verwendet wird. Bedenkt man, dass Züchtungsziele unter Nutzung technischen Wissens formuliert werden - wie dies etwa bei der Leistungszucht der Fall ist -, so lassen sich Züchtungsreihen progressiv als Optimierung aktueller Züchtungskollektive verstehen. Bezeichnen wir nun die - unter Nutzung nicht-evolutionären Wissens ${ }^{\mathrm{I3}}$ erstellten - Beschreibungen von Lebewesen - z. B. in biomechanischer Absicht - als Konstruktionen, so geben diese die »formalen « Bedingungen der Möglichkeit von Transformationen mit gesetztem Endglied an. Als Kriterien der Transformation können z. B. im Bereich der Technik einschlägige - Optimierungskriterien gelten, die ebenfalls in der irreflexiven progressiven Form auszuzeichnen sind. Nimmt man nun die Konstruktionen gegebener Lebewesen als Ausgangspunkt einer regressiven Reihe, so ergibt sich eine Abfolge von Antezedenten/Postsequenten-Konstruktionen. Die Transformationsreihe soll so gelesen werden, dass (in der Berichtreihenfolge) von einer Konstruktion nach Maßgabe von Rekonstruktionsprinzipien zur folgenden Konstruktion überzu-
Io Dies schließt gerade nicht aus, dass der als Beispiel angeführte Verlauf in immer stärkerem Maße der Handlungsverfügung unterworfen wird.

I I Übersieht man dies, so kommt es regelmäßig zu den mit dem Teleologie-, respektive Teleonomieproblem verbundenen methodologischen Engführungen. Diese lassen sich durch sprachkritische Rekonstruktion in der angezeigten Form vermeiden (näheres s. GUTMANN

(I996)).

I 2 Dazu im Einzelnen GutmanN (I996).

I3 Darunter können alle solchen biologischen Beschreibungen verstanden werden, die direkter laborgestützter Analyse zugänglich sind (etwa genetische, embryologische, cytologische, biochemische, physiologisch, taxonomische [sic]). 


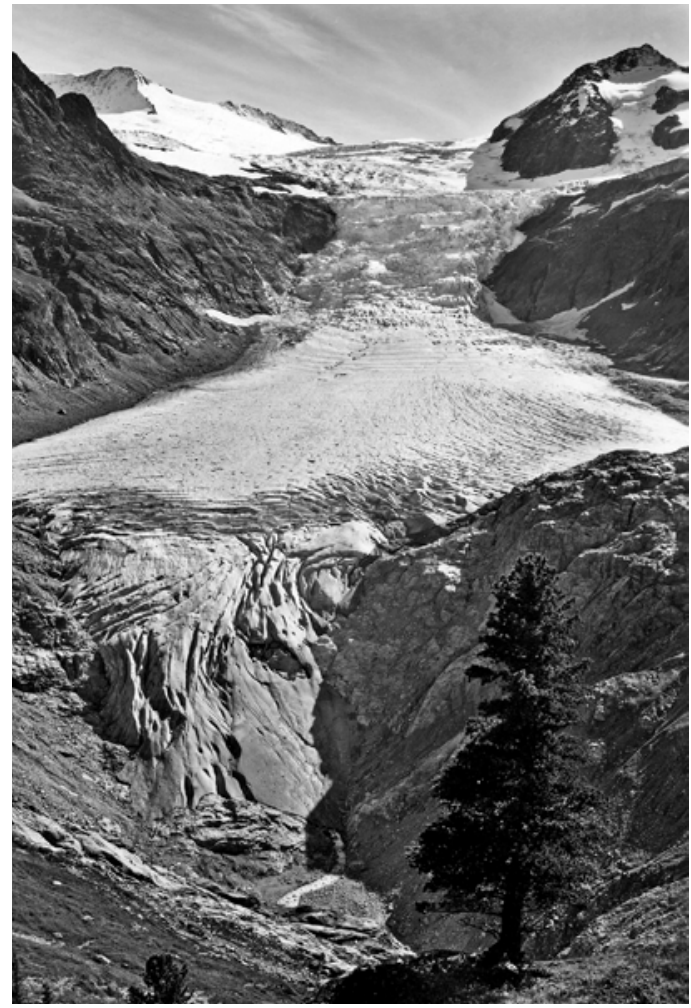

gehen sei. Als in der Zeitreihe von der Vergangenheit herkommend letzte Konstruktion gilt die End-Konstruktion, die allerdings als (methodisch) erste - nämlich an rezenten Lebewesen erstellt wurde. Als Rekonstruktionsprinzipien werden die Transformationsregeln bezeichnet, um die Umkehrung der Reihenfolge zu markieren; denn wie gesehen war die Verbesserung einer Maschine respektive eines Lebewesens (innerhalb der Züchtung, siehe oben) das gesetzte Handlungsziel, welches es zu realisieren galt.

Die »formalen « Bedingungen der Transformation lassen sich also an tatsächlichen Lebewesen unter modellgeleiteter Konstruktion ge- winnen. Damit kann relativ zum investierten Wissen $^{\mathrm{I} 4}$ ein Übergang von den rekonstruierten Zwischenstadien $\mathrm{zu}$ »möglichen Lebewesen « vollzogen werden. Der besondere Status der resultierenden "möglichen Lebewesen " ist am besten als »hypothetischer « zu bestimmen. Es handelt sich also nicht um eine einfache Umkehr des Sprachebenenwechsels, wie dies bei der Ausgangs- (i. e. End-)Konstruktion der Fall sein kann. Während die »Adäquatheit « der Konstruktionserstellung durch Überprüfung gewisser direkter Schlüsse etwa auf biomechanische Verhältnisse an rezenten Lebewesen stattfindet, entfällt diese Möglichkeit im hypothetischen Modus »möglicher Lebewesen«. Jeder rekonstruierten Konstruktion der Reihe wird also ein hypothetisches »Konstrukt « zur Seite gestellt. Diese können nun als »Prüfkriterien « für das Auffinden von Resten von Lebewesen dienen. Da Fossilien zunächst als » Reste « von Lebewesen zu deuten sind und unter Nutzung rezent-biologischen Wissens der Übergang von diesen Resten $\mathrm{zu}$ »möglichen « Lebewesen ${ }^{\mathrm{I} 5}$ gemacht werden muss, geben die aus den Transformationsreihen stammenden Formen eine Möglichkeit an die Hand, solche (paläontologischen) Wissensbestände zu Zwecken der Rekonstruktion zu verwenden. Dies ist z. T. bis ins Detail möglich, da ja den eingedeuteten Lebewesen auch »Teile« entsprechen - wiederum im hypothetischen Modus. An den eingedeuteten Lebewesen sowie ihren Teilen - deren Erstellung als eine Art »reverse engineering " oder Re-Konstruktion sensu verbis verstanden werden kann - und nicht an den Konstruktionen - erfolgt dann auch die weitere Modellierung etwa in physiologischer, populationsbiologischer oder ökologischer Form. Der Status evolutionstheoretischer Aussagen ist damit als "rekonstruktiver « zu bestimmen. "Zeit " kommt hier zunächst gar nicht ins Spiel; viel-

I 4 Das ist die hier relevante Ein-

schränkung. Es geht also im

Folgenden nicht um einen "Existenzbeweis « denkbarer Lebewesen, sondern nur darum, solche Lebewesen für möglich zu halten, die den in der Konstruktion angegebenen Kriterien entsprechen.

I 5 Dies gilt ebenfalls im hypothetischen Modus. 
mehr werden - ganz unabhängig von den genutzten Mitteln ${ }^{16}$ - ordinale Transformationsreihen erstellt, die erst in einem zweiten Schritt als empirische Zeitreihen umzudeuten sind. Zeit wäre also hier - obgleich Gegenstand der empirischen Forschung - zunächst methodisches Prius eines echten ordo succedendi. Der Ursprung einer in Frage stehenden Lebensform wäre dies nur in gewisser Hinsicht »zeitlich « im Sinne eines bestimmten Zeitpunktes oder Zeitraumes; er ist vielmehr methodischer Anfang wie Einheitspunkt der Rekonstruktion selbst, die uns erst die Rede von der Zeitlichkeit von Entwicklungsund Evolutionsvorgängen erlaubte.

\section{Ursprünge, Anfänge und Herkünfte}

In unserer Rekonstruktion erscheinen Evolutionstheorien als Defizienzformen historischer Theorien, die erst vor dem Hintergrund eines starken Verständnisses von Geschichte (das einen notwendigen Selbstbezug zum Erzählenden enthält) konstruiert werden können.

Wenn wir die Berichte über evolutionäre Rekonstruktionen der Form nach als »Ursprungserzählungen « ansprechen, dann ist zu bedenken, dass schon der Ausdruck »Ursprung « selbst mehrdeutig ist. Wir bezeichnen damit nämlich zunächst - und dies ist die übliche Verwendung einen (vergangenen) Zeitpunkt, an dem etwas in Existenz kam. Der Ur-Sprung wäre in dieser Lesart also ein letztlich empirisch bestimmbarer Zeitpunkt. Die empirische Bestimmung mag allerdings - etwa durch das Fehlen von Quellen (im Falle historischer Rekonstruktionen) oder durch die Unsicherheit der Situationsbeschreibung der Entstehung (z. B. in evolutionären Rekonstruktionen) - schwierig oder sogar unmöglich sein. In jedem Fall aber ist die Identifikation eines Ursprunges logisch zurückbezogen auf die Beschreibung des Gegenstandes, um dessen Entstehung es zu tun ist. Einem solchen empirischen Anfang, für den es charakteristisch ist, dass von ihm her eine Erzählung aufgebaut ist, die den Eindruck erweckt, der Erzähler der Berichte wäre persönlich zugegen gewesen, steht ein methodischer Anfang gegenüber. Der methodische Anfang umfaßt genau jene Beschreibung des Gegenstandes (wie etwa unsere oben als Konstruktionen beschriebenen Lebewesen), dessen Veränderung von der Gegenwart ausgehend rekonstruiert wird, sowie die Prinzipien der Rekonstruktion. Den Bericht über die Ergebnisse

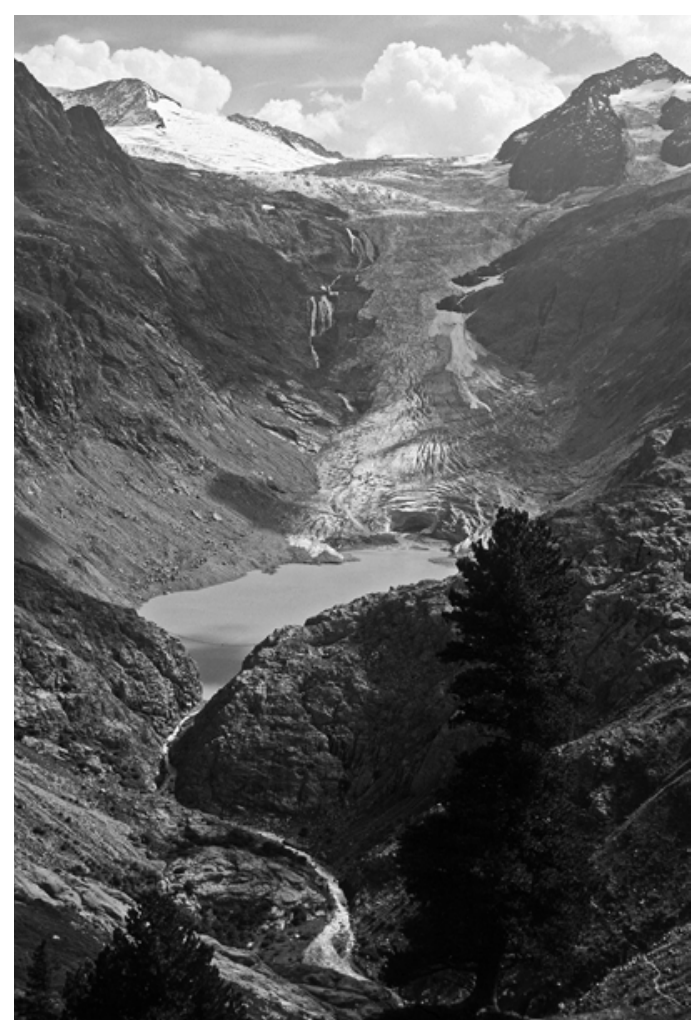

I6 Das heißt, diese Überlegungen gelten auch z. B. im Falle der phylogenetischen Methoden (s. o. unsere Darstellung zur molecular clock). 
dieser Rekonstruktion können wir als »Herkunftsbericht « bezeichnen, denn es wird über ein Herkommen von etwas in Frage Stehendem berichtet. Da der Gegenstand (im Prinzip beliebig viele) Beschreibungen ermöglicht, kann auch die Zahl der Herkünfte empirisch nicht abgeschlossen werden. Neben der Tatsache, dass Vergangenheit immer eine solche einer Gegenwart ist, besteht gerade hierin ein Grundproblem historischer Diskurse, das sich in ihrer grundsätzlichen Unabgeschlossenheit dokumentiert.

Bezüglich solcher herkünftigen Berichts-Erzählungen können wir nun auch eine weitere
Bedeutung des Ausdruckes »Ursprung « einführen. Der Ursprung bezeichnet die Einheitsstiftung der rekonstruierten Herkünfte. Seine Form ist die der Erzählung, die ihre Plausibilität aus den Herkunftsberichten speist. Die Kohärenz und Konsistenz des Ursprunges ist die Norm der Synthese der Herkünfte. Die gewonnene Herkunft verklärt sich zum Ursprung, wenn dieser mit einem zeitlichen Ereignis identifiziert wird.

\section{Literatur}

ARIstoteles: Physik, Bücher I - IV. Übersetzt von Zekl, Hamburg.

Augustinus: Confessiones. Übersetzt von Thimme, Düsseldorf, Zürich.

Cassirer, E. (I923): Philosophie der symbolischen Formen. Bd. I: Die Sprache (I988).

CAssirer, E. (I924): Philosophie der symbolischen Formen. Bd. 2: Das mythische Denken (1987).

CAssirer, E. (I929): Philosophie der symbolischen Formen. Bd. 3: Phänomenologie der Erkenntnis (I990).

Conroy, G. C. (I997): Reconstructing Human origins, New York, London.

Graur, D. und Li, Wen-Hsiung (2000): Fundamentals of Molecular Evolution, Sunderland, Massachusetts.

Gutmann, M. (I996): Die Evolutionstheorie und ihr Gegenstand - Beitrag der Methodischen Philosophie zu einer konstruktiven Theorie der Evolution, Berlin.

GutmanN, M. (2002): Human Cultures' Nature, in: Grunwald, A., Gutmann, M. und NeumanNHeld, E. M. (Hg.), On Human
Nature. Anthropology: Biological and Philosophical Foundation, Berlin, 195-240.

Gutmann, M. und Weingarten, M. (200I): Die Bedeutung von Metaphern für die biologische Theorienbildung, in: Deutsche Zeitschrift für Philosophie 49/4, 549566.

Janich, P. (I980): Die Protophysik der Zeit. Frankfurt a. M.

JaNICH, P. (I988): Zeit, Bewegung, Handlung, in: Rudolph, E. (Hg.), Studien zur Zeitabhandlung des Aristoteles, Stuttgart, I 67-I92.

Janich, P. (I989): Euklids Erbe, München.

Janich, P. (I996): Die Konstitution der Zeit durch Handeln und Reden. Kodikas/Code, Vol. I9 Nr. I/2, Jan./Jun. I996, I33-I47.

Janich, P. (I997): Kleine Philosophie der Naturwissenschaften, München.

JANICH, P. (I999): Die Naturalisierung der Natur, Sitzungsber. Wiss. Ges. Johann Wolfgang Goethe-Univ., Frankfurt a. M., Stuttgart.

KuTsChera, U. (2006): Evolutionsbiologie, Stuttgart.

\section{Mathias Gutmann}

Levit, G. S., Meister, K. und HossFELD, U. (2005): Alternative Evolutionstheorien, in: KroHs, U. und Toepfer, G. (Hg.), Philosophie der Biologie, Frankfurt a. M., 267-286.

LoRenZ, K. (I973): Die Rückseite des Spiegels, München.

Mletzko, H.-G. und Mletzko, I. (I985): Biorhythmik, Wittenberg.

Oeser, E. (1987): Evolutionäre Wissenschaftstheorie, in: LÜTTERSFELD, W. (Hg.), Transzendentale oder evolutionäre Erkenntnistheorie?, Darmstadt, 5 I-63.

Simpson, G. G. (1963): Biology and the nature of science, Science I39, $8 \mathrm{I}-88$.

Tembrock, G., Siegmund, R. und Nichelmann, M. (Hg.) (I986): Verhaltensbiologie. Internationales Symposion I983, Berlin.

UеXкÜLL, J. v. (I973): Theoretische Biologie, Frankfurt a. M.

VolLmer, G. (I987): Evolutionäre Erkenntnistheorie, Stuttgart.

WinfreE, A. T. (I986): Biologische Uhren, Heidelberg. 